CHRONIC OBSTRUCTIVE PULMONARY DISEASE

\title{
Effect of oxygen on recovery from maximal exercise in patients with chronic obstructive pulmonary disease
}

\author{
N J Stevenson, P M A Calverley
}

Thorax 2004;59:668-672. doi: 10.1136/thx.2003.014209

See end of article for authors' affiliations

\section{Correspondence to:}

Professor P M A Calverley, Clinical Science Centre, University Hospital

Aintree, Liverpool L9 7AL, UK; pmacal@liverpool.ac uk

Received 6 August 2003 Accepted 4 March 2004
Background: The effects of oxygen on recovery from exercise in patients with chronic obstructive pulmonary disease (COPD) are not clearly known. A study was undertaken to determine whether oxygen given after maximal exercise reduced the degree of dynamic hyperinflation and so reduced the perception of breathlessness.

Methods: Eighteen patients with moderate to severe COPD performed maximal symptom limited exercise on a cycle ergometer. During recovery they received either air or oxygen at identical flow rates in a randomised, single blind, crossover design. Inspiratory capacity, breathing pattern data, dyspnoea intensity, and leg fatigue scores were collected at regular intervals during recovery. At a subsequent visit patients underwent a similar protocol but with a face mask in situ to eliminate the effects of instrumentation. Results: When oxygen was given the time taken for resolution of dynamic hyperinflation was significantly shorter (mean difference between air and oxygen 6.61 (1.65) minutes (95\% Cl 3.13 to 10.09), $p=0.001$ ). Oxygen did not, however, reduce the perception of breathlessness during recovery nor did it affect the time taken to return to baseline dyspnoea scores in either the instrumented or non-instrumented state (mean difference 2.11 (1.41) minutes (95\% Cl -0.88 to 5.10$), p=0.15)$.

Conclusions: Oxygen reduces the degree of dynamic hyperinflation during recovery from exercise but does not make patients feel less breathless than breathing air. This suggests that factors other than lung mechanics may be important during recovery from exercise, or it may reflect the cooling effect of both air and oxygen.
B reathlessness is the most disabling symptom associated with chronic obstructive pulmonary disease (COPD) and its relief is an important therapeutic goal. ${ }^{1}$ It is usually provoked by exertion and the resultant reduction in exercise capacity is itself a major determinant of impaired health status in COPD. ${ }^{23}$

Short acting bronchodilator drugs can reduce dyspnoea and increase exercise tolerance in $\mathrm{COPD},{ }^{45}$ principally by limiting the increase in end-expiratory lung volume that occurs in this disease. ${ }^{6}$ This is most evident in more severe disease, occurs during self-paced as well as cycle exercise, and appears to be secondary to tidal expiratory flow limitation present before exercise or occurring during it. ${ }^{8}$

Breathing supplementary oxygen during exercise increases exercise duration and reduces the intensity of dyspnoea at any workload. ${ }^{10}$ These effects occur independently of the initial arterial oxygen tension and are more evident at higher flow rates. ${ }^{11}$ Reduction in the degree of dynamic hyperinflation secondary to a fall in minute ventilation when breathing oxygen explains most of this improvement in patients with COPD.

Much less is known about the physiological basis for "as needed" oxygen therapy in the treatment of breathlessness occurring at rest or after exercise. Patients with severe COPD are often advised to use oxygen after exercise to increase the rate of resolution of their dyspnoea. ${ }^{12}$ However, the evidence to support this is conflicting with some studies in favour ${ }^{13}$ but others against ${ }^{14} 1^{15}$ there being any clinically important benefits.

In this study we hypothesised that giving oxygen at high flow rates to patients with COPD after exercise would reduce their degree of dynamic pulmonary hyperinflation and change their breathing pattern when ventilation was highest in the first 5 minutes after exercise ceased. As a result, the overall speed of resolution of their breathlessness would be increased when breathing oxygen. We anticipated that these changes would be most evident in patients with tidal flow limitation at rest where, presumably, the degree of dynamic lung volume change would be greatest. To test this we conducted a randomised, single blind, crossover trial comparing oxygen and room air given at identical flow rates and measured inspiratory capacity, breathing pattern, and dyspnoea intensity as exercise resolved. To exclude any effect of the physiological instrumentation, patients repeated the same protocol without the mouth piece and nose clip but breathing air or oxygen from a face mask, as would occur in normal clinical practice.

\section{METHODS}

\section{Subject recruitment}

Patients aged 40-79 years with stable COPD were randomly recruited from the respiratory outpatient department. COPD was defined using BTS/ERS criteria and all patients had a forced expiratory volume in 1 second $\left(\mathrm{FEV}_{1}\right)$ of $<80 \%$ predicted and a ratio of $\mathrm{FEV}_{1}$ to forced vital capacity (FVC) of $<70 \%$. Patients were excluded if they had an exacerbation of COPD, if there had been any change in their medication in the 4 weeks before the study, or if they were receiving long term domiciliary oxygen therapy. Patients unable to perform exercise testing-for example, as a result of neuromuscular problems or peripheral vascular disease-and those in whom exercise testing was contraindicated-for example, patients

Abbreviations: $\mathrm{FEV}_{1}$, forced expiratory volume in 1 second; $\mathrm{FRC}$, functional residual capacity; FVC, forced vital capacity; IC, inspiratory capacity; MIP, maximal inspiratory capacity; MEP, maximal expiratory capacity; NEP, negative expiratory pressure; TFL, tidal expiratory flow limitation; $\mathrm{TLC}$, total lung capacity; $\mathrm{VE}$, minute ventilation; $\mathrm{VO}_{2}$ max, maximal oxygen consumption; $\mathrm{V}_{\mathrm{CO}_{2}}$ max, maximal carbon dioxide production; $\mathrm{VT}$, tidal volume 
with acute coronary syndrome-were not studied. Study approval was granted by the local research ethics committee and written informed consent was obtained.

\section{Pulmonary function testing}

Spirometric tests were performed using a rolling seal spirometer (MedGraphics 1070, Medical Graphics, St Paul, MN, USA) and met established British Thoracic Society standards. The highest value for $\mathrm{FEV}_{1}$ and FVC from three reproducible tracings was used. Inspiratory capacity (IC) was calculated as the volume inspired from the patients' endexpiratory lung volume to total lung capacity (TLC). Satisfactory technique and reproducibility of IC manoeuvres for each subject were established initially under resting conditions and conformed to the methods described by other workers. ${ }^{6}$ TLC was assumed to be constant throughout the exercise and recovery periods. All patients were familiarised with the exercise testing protocol before the first measurements were made. When IC was measured during exercise, the patients were warned that the measurement was about to be made a few breaths beforehand and then told: "At the end of the next normal breath, take a deep breath all the way in", together with verbal encouragement to make a maximal effort.

Expiratory flow limitation was assessed by applying negative expiratory pressure (NEP) during tidal breathing as previously described. ${ }^{16}$ Expiratory flow limitation was deemed to be present when the application of NEP did not result in an increase in expiratory flow during most of expiration. Maximal inspiratory and expiratory mouth pressures (MIP and MEP) measured at functional residual capacity (FRC) and TLC, respectively, were assessed with a standard mouthpiece and pressure manometer (P K Morgan, Chatham, Kent, UK). Tidal breathing pattern was recorded with the patient breathing normally through a pneumotachograph (MedGraphics 1070, Medical Graphics). Computer software was used to derive timing (frequency, TI, TE, Ttot), tidal volume (VT), mean inspiratory and expiratory flow (VT/ $\mathrm{TI}, \mathrm{VT} / \mathrm{Te})$, and expired minute ventilation (VE). Oxygen saturation $\left(\mathrm{SaO}_{2}\right)$ was measured using a pulse oximeter attached to the pinna.

\section{Exercise testing}

Patients exercised on an electrically braked cycle ergometer wearing a noseclip and breathing through a mouthpiece. Ventilatory data and its derivatives were recorded breath by breath throughout the test as was oxygen consumption $\left(\mathrm{VO}_{2}\right)$ using a fuel cell and carbon dioxide production $\left(\mathrm{VCO}_{2}\right)$ with an infrared analyser. Resting data, including the Borg symptom scores for breathlessness and leg fatigue, were recorded for 2 minutes before exercise. From $0 \mathrm{~W}$ the workload was increased by $10 \mathrm{~W}$ every 2 minutes until symptom limitation. Borg scores were recorded every 2 minutes during exercise.

\section{Evaluation of dyspnoea and leg effort}

Dyspnoea was assessed by the response to the question: "How breathless do you feel?" and leg fatigue by the question: "How tired do your legs feel?". Patients were familiarised with the modified Borg score before testing. They were asked to point to the Borg scale corresponding to their current symptom intensity at rest, during exercise, and during recovery.

\section{Study design}

Patients attended on two occasions separated by at least l week. At each visit resting Borg scores and oxygen saturation on air were recorded. Baseline lung function testing was performed in the same sequence for each patient at both visits and involved the measurement of flow limitation using the NEP technique, MIP and MEP, tidal breathing analysis, IC, and spirometry. Patients were advised to avoid caffeine and heavy meals for 4 hours before testing. All machines were accurately calibrated before the test sequence.

At each visit patients then performed a maximal cardiorespiratory exercise test. As soon as exercise stopped, patients randomly received either air or oxygen $\left(\mathrm{FiO}_{2} 0.4\right)$ in a single blind crossover fashion. At one visit the patient remained instrumented during recovery while at the other visit the mouthpiece and noseclips were replaced with a Venturi mask at a flow rate of $10 \mathrm{l} / \mathrm{min}$. Patients were allowed to rest for a minimum of 45 minutes between exercise tests. When the patients remained instrumented during recovery, Borg score, tidal breathing pattern and IC were recorded every 3 minutes for 15 minutes. When patients were non-instrumented during recovery, Borg scores were recorded every minute for 15 minutes. The order of the visits was randomised in each patient, so patients were randomised to either the instrumented or non-instrumented state at the first visit with crossover at the second visit. Oxygen was administered in random order at both visits.

The study was powered on the assumption that oxygen therapy would produce a difference of $200 \mathrm{ml}$ in IC by 4 minutes after exercise. This figure was selected as being equivalent to the minimum difference in IC seen with isotime comparisons when patients were receiving $60 \%$ oxygen. ${ }^{17} \mathrm{~A}$ study of 13 patients would have a $90 \%$ power to detect such a difference. We did not power the trial on time to recovery as we had insufficient prior data to do this, although we anticipated that a significant change in IC would affect the recovery time. Likewise, data about the speed of symptomatic recovery in this patient group was lacking and we accepted that any change in symptom recovery time that achieved statistical significance would be of clinical interest in a study of this size.

\section{Statistical analysis}

Descriptive data are expressed as mean (SD) while other statistical data are expressed as mean (SE). Single paired comparisons were performed using Student's $t$ tests and nonparametric data were analysed using the Wilcoxon rank sum test. Repeated measures data were analysed using summary measures over time which are expressed as mean (SE) with 95\% confidence intervals (CI). When data involved more than one comparison, ANOVA was used to assess the significance of differences between the groups, a p value of $<0.05$ being accepted as significant for all analyses.

\section{RESULTS}

\section{Subjects}

Fifty four patients were screened of whom 38 met the study criteria and 18 agreed to participate. One patient declined to attend for visit 2, during which recovery was randomly allocated to be non-instrumented. There were no statistical differences in baseline pulmonary function between visits (table 1). No patient was hypoxaemic at rest, although six patients desaturated during the maximal cardiorespiratory exercise test-the lowest $\mathrm{SaO}_{2}$ was $88 \%$ (range 88-96\%) and the longest period of desaturation was 6 minutes. Patients who desaturated with exercise had a lower baseline FEV $_{1}$ than those who maintained their $\mathrm{SaO}_{2}$ constant when exercising $(0.73(0.09) \mathrm{l} \vee 1.26(0.09) \mathrm{l} ; \mathrm{p}=0.01)$. Four of the six patients who desaturated also exhibited tidal flow limitation at rest.

The presence of desaturation during exercise did not influence the subsequent results of oxygen treatment.

At each of the four cardiorespiratory exercise tests the mean (SD) duration of exercise (8.02 (0.39) minutes) and 


\begin{tabular}{|c|c|}
\hline No of patients & 18 \\
\hline $\operatorname{Sex}(M: F)$ & $12: 6$ \\
\hline Age (years) & $61.2(4.4)$ \\
\hline $\mathrm{FEV}_{1}$ (litres) & $1.08(0.42)$ \\
\hline $\mathrm{FEV}_{1}$ (\% predicted) & 40.28 (15.93) \\
\hline IC (litres) & $2.17(0.64)$ \\
\hline IC (\% predicted) & $86.88(25.27)$ \\
\hline $\operatorname{MIP}\left(\mathrm{cm} \mathrm{H}_{2} \mathrm{O}\right)$ & 70.18 (16.47) \\
\hline $\operatorname{MEP}\left(\mathrm{cm} \mathrm{H}_{2} \mathrm{O}\right)$ & $105.28(21.98)$ \\
\hline $\mathrm{SaO}_{2}(\%)$ & $95.94(1.66)$ \\
\hline $\begin{array}{l}\text { Resting Borg breathlessness } \\
\text { score }\end{array}$ & $0.84(0.87)$ \\
\hline Resting Borg leg score & $1.0(1.12)$ \\
\hline TFL & $7 / 18$ \\
\hline \multicolumn{2}{|c|}{$\begin{array}{l}\text { Values given are mean (SD) of visits } 1 \text { and } 2 \text {. } \\
\mathrm{FEV}{ }_{1} \text {, forced expiratory volume in } 1 \text { second; IC, inspiratory } \\
\text { capacity; MIP, maximal inspiratory capacity; } M E P \text {, maxima } \\
\text { expiratory capacity; TFL, tidal expiratory flow limitation. }\end{array}$} \\
\hline
\end{tabular}

maximal workload (37.5 (3.98) W) were not significantly different (table 2). In addition, there was no statistical difference between tests in the maximum Borg breathlessness and leg scores achieved (mean (SD) 5.30 (2.04) and 5.30 (1.94), respectively).

Seven patients had expiratory flow limitation during tidal breathing at rest in the seated position. $\mathrm{FEV}_{1}$ did not differ significantly between patients with and without flow limitation (0.88 (0.13) l v l.21 (0.14) l; p=0.09). In addition, Borg breathlessness scores at rest did not differ significantly between patients with and without flow limitation $(0.75$ (0.44) $v 1.27(0.28) ; \mathrm{p}=0.31)$. In most patients $(\mathrm{n}=16)$ IC was lower when measured 1 minute after the end of exercise, reflecting dynamic hyperinflation. The two patients who did not show evidence of hyperinflation at this time were not flow limited at rest; however, their baseline $\mathrm{FEV}_{1}$ was not statistically different from that of patients who exhibited dynamic hyperinflation.

\section{Ventilation, breathing pattern, and lung mechanics after exercise}

The mean (SD) resting VE at both visits was 14.46 (5.28) l/ min. During maximal exercise this increased to 23.68 (4.84) $\mathrm{l} / \mathrm{min}$. The mean (SE) difference in VE over the recovery period between air and oxygen was 0.79 (0.58) l/ $\min (95 \% \mathrm{CI}-0.71$ to 2.29$) ; \mathrm{p}=0.24$ (fig 1). Mean (SE) baseline VT was 0.75 (0.05) l which increased to 0.89 (0.02) l at maximal exercise. Breathing frequency was 21.33 (1.18) breaths/min at baseline, peaking at 28.53 (0.70) breaths/min at maximum exercise. Neither VT nor

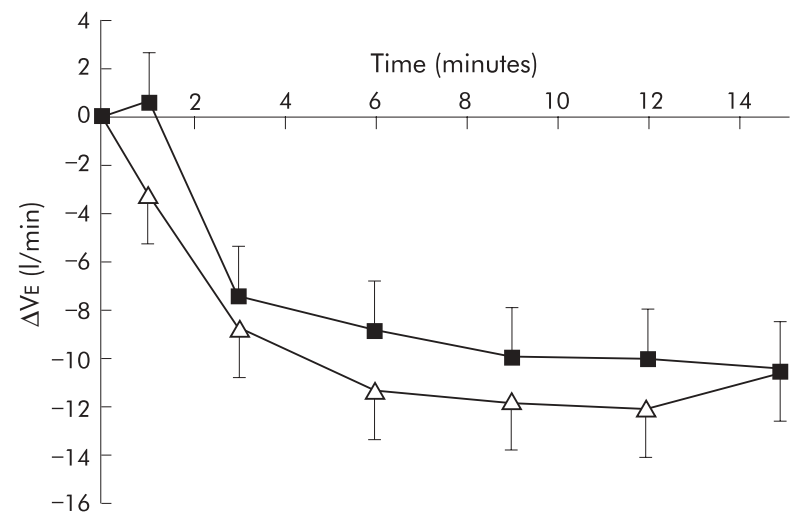

Figure 1 Change in minute ventilation $\left(V_{E}\right)$ over time after the end of exercise when breathing oxygen (open symbols) and breathing air (solid symbols). No significant difference in VE was seen between the gas mixtures.

breathing frequency was affected by oxygen at any time during recovery.

At baseline, mean (SD) IC at both visits was $2.22(0.62) \mathrm{l}$. One minute into the recovery period the mean (SD) IC was 2.01 (0.56) l. Patients with resting expiratory tidal flow limitation, as assessed by the NEP technique, did not have greater dynamic hyperinflation at this point after exercise. By 4 minutes after exercise the IC in the oxygen treated patients was significantly less than in those breathing air $(\mathrm{p}<0.01$; fig 2). The mean (SE) difference in IC during recovery between air and oxygen was $-0.27(0.13) \mathrm{l}(95 \% \mathrm{CI}-0.60$ to $0.07) ; p=0.07$ ). The time taken for resolution of dynamic hyperinflation was significantly shorter when oxygen was administered (mean difference between treatments 6.61 ( 1.65 ) minutes (95\% CI 3.13 to 10.09 ); $p=0.001$ ). This was true for patients with and without tidal flow limitation at rest.

The mean (SD) Borg breathlessness score at rest in all four tests was $0.87(1.02)$, rising to $5.30(2.04)$ at maximal exercise. Breathlessness scores fell with recovery. There was no statistical difference in Borg scores at any time during recovery between oxygen and air irrespective of the presence of instrumentation, nor was the time to return to the preexercise level of breathless affected by the gas inhaled (fig 3). The mean (SE) difference in Borg breathlessness scores over the recovery period when instrumented was $0.009(0.12)$, ( $95 \%$ CI -0.21 to 0.39 ); $p=0.47$. When non-instrumented, the mean (SE) difference in Borg score over time was -0.14 (0.007), $(95 \% \mathrm{CI}-0.31$ to 0.00$) ; \mathrm{p}=0.10$. In the instrumented

\begin{tabular}{|c|c|c|c|c|}
\hline Test & $\begin{array}{l}\text { Air with } \\
\text { mouthpiece }\end{array}$ & $\begin{array}{l}\text { Oxygen with } \\
\text { mouthpiece }\end{array}$ & $\begin{array}{l}\text { Air with } \\
\text { mask }\end{array}$ & $\begin{array}{l}\text { Oxygen with } \\
\text { mask }\end{array}$ \\
\hline Resting Borg breathlessness score & $0.97(0.28)$ & $0.75(0.25)$ & $1.03(0.26)$ & $0.74(0.21)$ \\
\hline Resting Borg leg score & $1.06(0.31)$ & $1.03(0.25)$ & $0.94(0.27)$ & $0.97(0.26)$ \\
\hline Exercise duration (minutes) & $8.16(0.96)$ & $7.07(0.87)$ & $8.18(0.95)$ & $8.65(0.98)$ \\
\hline $\begin{array}{l}\text { Maximal exercise Borg } \\
\text { breathlessness score }\end{array}$ & $5.36(0.55)$ & $5.17(0.51)$ & $5.26(0.49)$ & $5.41(0.51)$ \\
\hline Maximal exercise Borg leg score & $5.56(0.47)$ & $5.19(0.39)$ & $5.00(0.50)$ & $5.44(0.52)$ \\
\hline Maximal workload (W) & $37.22(5.53)$ & $32.78(5.47)$ & $38.23(5.30)$ & $41.18(5.55)$ \\
\hline $\mathrm{VO}_{2} \max (\mathrm{l} / \mathrm{min})$ & $0.97(0.09)$ & $0.94(0.08)$ & $0.94(0.08)$ & $0.91(0.07)$ \\
\hline $\mathrm{VCO}_{2} \max (1 / \mathrm{min})$ & $0.85(0.08)$ & $0.82(0.08)$ & $0.83(0.07)$ & $0.82(0.07)$ \\
\hline
\end{tabular}

Values are mean (SE).

$\mathrm{VO}_{2} \mathrm{max}$, maximal oxygen consumption; $\mathrm{V}_{\mathrm{CO}_{2}} \mathrm{max}$, maximal carbon dioxide production.

Statistical analysis between groups was performed using ANOVA. All $p$ values $>0.05$. 


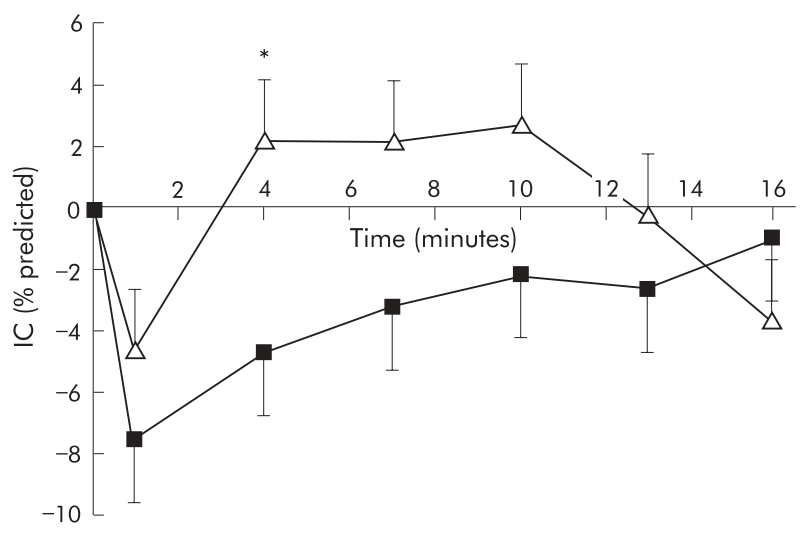

Figure 2 Change in inspiratory capacity (IC) expressed as a percentage of predicted value for age and height during recovery from exercise when breathing oxygen (open symbols) and breathing air (solid symbols). IC fell after exercise and returned to baseline values during recovery. It was significantly greater at 4 minutes when breathing oxygen than when breathing air; ${ }^{*} p<0.01$. The rate of recovery of IC was more rapid during oxygen breathing although overall the difference in IC at all time points was not significantly different.

patients the time taken to return to the baseline dyspnoea score was not significantly different when breathing oxygen (mean (SE) difference between air and oxygen 2.11 (1.41) minutes (95\% CI -0.88 to 5.10); $\mathrm{p}=0.15)$. Similarly, when patients were non-instrumented there was no difference in the time to symptomatic recovery between gases (mean (SE) difference between air and oxygen 0.47 (0.46) minutes ( $95 \% \mathrm{CI}-0.51$ to 1.45$) ; \mathrm{p}=0.32$ ). However, the time take for the dyspnoea score to return to the baseline levels was greater when breathing air through the mouthpiece than when it was administered from a face mask (mean (SE) difference 3.94 (1.77) minutes (95\% CI 0.20 to 7.69$) ; \mathrm{p}=0.04$ ).

The mean (SD) Borg score for leg effort was 1.00 (1.12) at rest and increased to $5.30(1.94)$ at maximal exercise. Leg fatigue scores were not statistically different at any point during recovery when breathing oxygen. In addition, the time taken to return to baseline scores did not differ when oxygen or air was administered. The mean (SE) difference between air and oxygen in the time taken to return to baseline was 0.65 (1.07) minutes (95\% CI -1.62 to 2.91 ); $\mathrm{p}=0.55$.

\section{DISCUSSION}

Although there is good evidence for the clinical benefit of oxygen administration during exercise in patients with COPD, equivalent data supporting the use of oxygen to help breathlessness resolve more rapidly when exercise stops are scanty. ${ }^{18}$ Despite this, most cylinder oxygen in the UK is prescribed for this purpose and to control acute dyspnoea, an indication where there is some experimental evidence of effectiveness. ${ }^{1920}$ Since the completion of our study further data have been published showing that oxygen after exercise does not appear to influence the rate of symptomatic recovery. ${ }^{15}{ }^{21}$ Our data using a more robust trial design suggest that supplementary oxygen does reduce dynamic hyperinflation more rapidly than breathing room air after exercise stops. However, this does not translate into a significant reduction in the degree of dyspnoea at any time after exercise, nor does it influence the rate at which symptoms resolve.

In this study we used a standardised progressive exercise protocol to produce the same level of breathlessness before giving either air or oxygen, something not always done in
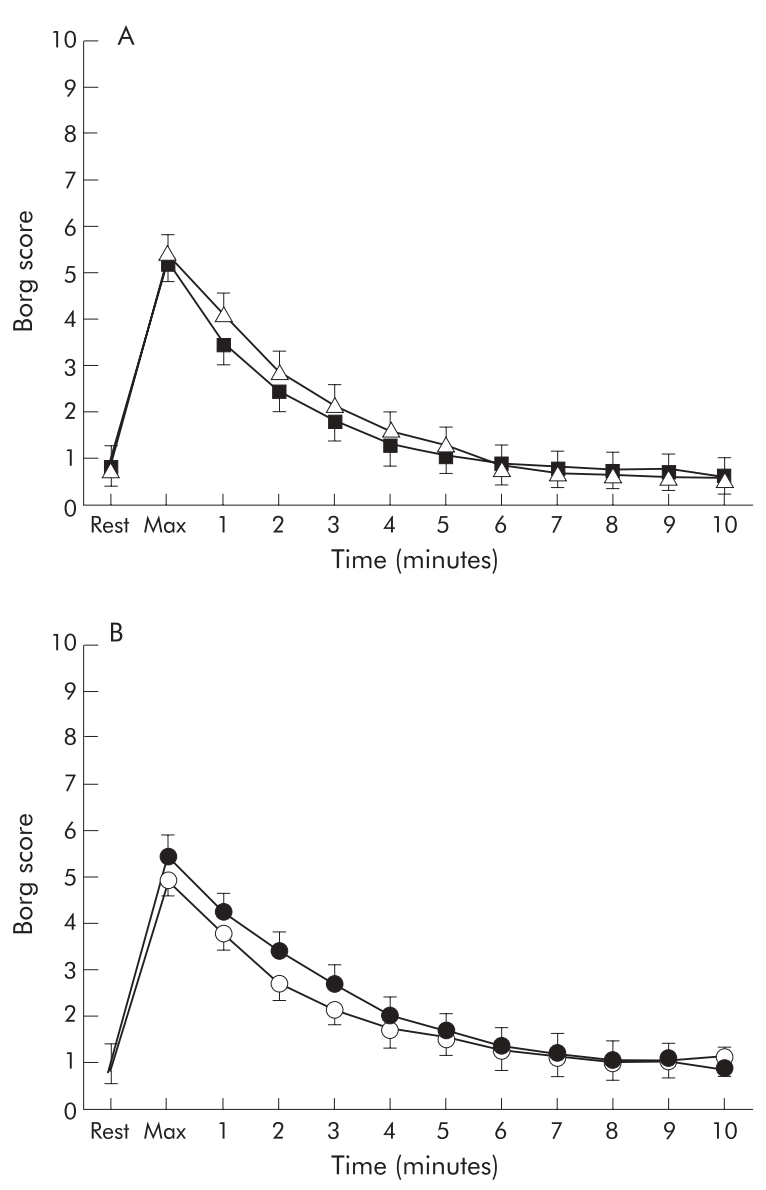

Figure 3 Borg score for breathlessness before and after exercise in (A) patients breathing oxygen (open symbols) or air (solid symbols) with nose clip and mouthpiece and (B) patients breathing from a face mask.

previous studies. The duration of exercise and degree of metabolic load incurred were similar in each test. The degree of oxygen desaturation observed during or at the end of exercise did not influence the subsequent symptomatic response to oxygen breathing. We measured IC before and soon after exercise ended to allow time for the inspired gas to have an effect. As a result, our IC values were somewhat lower than those recorded at maximum exercise or immediately after stopping walking. ${ }^{72}$ As our purpose was to compare the effect of oxygen and air on dynamic inflation, we did not relate these data to peak values during exercise. The need to obtain technically satisfactory measurements and to record symptom intensity determined the timing of the measurements during the recovery period. In practice, most of the change in dynamic hyperinflation and symptoms occurred 3-6 minutes after exercise ended.

Breathing oxygen ( $\mathrm{FiO}_{2}$ 0.4, flow $10 \mathrm{l} / \mathrm{min}$ ) did not significantly affect either ventilation or breathing pattern during the recovery period. The apparent early difference in ventilation seen in fig 1 was not accompanied by consistent changes in ventilation at other time points during recovery. The degree of dynamic hyperinflation was different by 4 minutes after exercise and this probably explains the more rapid resolution of this phenomenon during oxygen breathing. As different individuals recover at different times after exercise ends, it is not surprising that there is more "noise" in the latter measurements and it is proportionately harder to demonstrate changes in related variables such as ventilation and breathing pattern than is the case during endurance 
exercise testing. Nonetheless, oxygen does reduce the degree of dynamic hyperinflation more rapidly but, in contrast to the situation during exercise, this does not appear to be the major determinant of dyspnoea.

\section{Perception of breathlessness and leg effort}

Dynamic hyperinflation occurs more frequently in patients with expiratory flow limitation at rest $^{23}$ and can be reduced by oxygen breathing at rest. ${ }^{20}$ We did not find a clear association with tidal expiratory flow limitation and the presence of dynamic hyperinflation 1 minute after exercise in our patients. This may reflect the onset of flow limitation during exercise as noted by others. ${ }^{24}$ In our patients the presence of resting expiratory flow limitation did not identify a subgroup more responsive to supplementary oxygen.

The failure of oxygen to affect dyspnoea intensity reflects the different conditions present after exercise compared with those during exercise. When patients with COPD exercise there is a progressive rise in the respiratory drive to breathe due to metabolic $\mathrm{CO}_{2}$ production, increasing blood lactate concentrations, and changes in arterial blood gas tensions. In these circumstances any factor that reduces ventilatory drive-such as supplementary oxygen-will modify the breathing pattern, improve lung emptying, reduce dynamic hyperinflation, and lessen dyspnoea. After exercise the metabolic drive to breathing declines exponentially whatever gas mixture is inhaled at a speed that is influenced by many factors including the redistribution of regional blood flow, lactate metabolism, and any co-existing cardiac dysfunction. Although changes in dynamic lung volume still occur, their relative importance is less than the declining central respiratory drive and does not appear to significantly affect the intensity of dyspnoea.

Although oxygen did not influence the intensity or rate of resolution of symptoms, the presence of a mouthpiece and noseclip did. This may explain some of the differences in recovery time from exercise previously reported in the literature. We chose high gas flow rates to ensure that the optimal effect on dyspnoea was achieved ${ }^{11}$ but, in doing so, may have provided some relief from dyspnoea with both gas mixtures, the facial and upper airway cooling effect being known to reduce dypnoea during exercise in COPD. ${ }^{25}$

Our data help to explain why oxygen has less effect as symptomatic treatment than might be anticipated from its known effects during exercise, and support recently reported data on this topic. ${ }^{15}$ Administration of oxygen or compressed air may be a useful way of providing a source of cooling gas flow, but other cheaper and more convenient methods of doing this are worth exploring in future trials. The routine use of oxygen to aid recovery of symptoms after exercise does not appear to be warranted.

\section{Authors' affiliations}

N J Stevenson, P M A Calverley, Clinical Science Centre, University Hospital Aintree, Liverpool L9 7AL, UK

\section{REFERENCES}

1 Pauwels RA, Buist AS, Calverley PMA, et al. Global strategy for the diagnosis, management and prevention of chronic obstructive pulmonary disease. Am J Respir Crit Care Med 2001;163:1256-76.

2 Jones PW, Quirk FH, Baveystock CM, et al. A self-complete measure of health status for chronic airflow limitation. The St George's Respiratory Questionnaire. Am Rev Respir Dis 1992;145:1321-7.

3 Dowson U, Newall C, Guest PJ, et al. Exercise capacity predicts health status in alpha(1)-antitrypsin deficiency. Am J Respir Crit Care Med $2001 ; 163: 936-41$

4 Hay JG, Stone P, Carter J, et al. Bronchodilator reversibility, exercise performance and breathlessness in stable chronic obstructive pulmonary disease. Eur Respir J 1992;5:659-64.

5 O'Donnell DE, Lam M, Webb KA. Spirometric correlates of improvement in exercise performance after anticholinergic therapy in chronic obstructive pulmonary disease. Am J Respir Crit Care Med 1999;160:542-9.

6 O'Donnell DE, Webb KA. Exertional breathlessness in patients with chronic airflow limitation. The role of lung hyperinflation. Am Rev Respir Dis 1993; 148:1351-7.

7 Marin JM, Carrizo SJ, Gascon M, et al. Inspiratory capacity, dynamic hyperinflation, breathlessness, and exercise performance during the 6-minutewalk test in chronic obstructive pulmonary disease. Am J Respir Crit Care Med 2001; 163:1395-9.

8 Boni E, Corda L, Franchini D, et al. Volume effect and exertional dyspnoea after bronchodilator in patients with COPD with and without expiratory flow limitation at rest. Thorax 2002;57:528-32.

9 Calverley PM, Leggett RJ, Flenley DC. Carbon monoxide and exercise tolerance in chronic bronchitis and emphysema. BMJ 1981;283:878-80.

10 O'Donnell DE, Bain DJ, Webb KA. Factors contributing to relief of exertional breathlessness during hyperoxia in chronic airflow limitation. Am J Respir Crit Care Med 1997:155:530-5.

11 Leach RM, Davidson AC, Chinn S, et al. Portable liquid oxygen and exercise ability in severe respiratory disability. Thorax. 1992;47: 781-9; erratum, 1993;48:192.

12 COPD Guidelines Group of the Standards of Care Committee of the BTS. BTS guidelines for the management of chronic obstructive pulmonary disease. Thorax 1997;52(Suppl 5):S1-28.

13 Killen JW, Corris PA. A pragmatic assessment of the placement of oxygen when given for exercise induced dyspnoea. Thorax 2000;55:544-6.

14 Marques-Magallanes JA, Storer TW, Cooper CB. Treadmill exercise duration and dyspnea recovery time in chronic obstructive pulmonary disease: effects of oxygen breathing and repeated testing. Respir Med 1998;92:735-8.

15 Nandi K, Smith AA, Crawford A, et al. Oxygen supplementation before or after submaximal exercise in patients with chronic obstructive pulmonary disease. Thorax 2003;58:670-3.

16 Hadcroft J, Calverley PM. Alternative methods for assessing bronchodilator reversibility in chronic obstructive pulmonary disease. Thorax 2001;56:713-20

17 O'Donnell DE, D'Arsigny C, Webb KA. Effects of hyperoxia on ventilatory limitation during exercise in advanced chronic obstructive pulmonary disease. Am J Respir Crit Care Med 2001;163:892-8.

18 Rudolf M, Wedzicha JA, Calverley PMA, et al. Domiciliary oxygen therapy services: clinical guidelines and advice to prescribers. London: Royal College of Physicians, 1999:1-49.

19 Swinburn CR, Mould H, Stone TN, et al. Symptomatic benefit of supplemental oxygen in hypoxemic patients with chronic lung disease. Am Rev Respir Dis $1991 ; 143: 913-5$.

20 Liistro G, Stanescu D, Rodenstein D, et al. Reassessment of the interruption technique for measuring flow resistance in humans. J Appl Physiol 1989;67:933-7.

21 Lewis CA, Eaton TE, Young $P$, et al. Short-burst oxygen immediately before and after exercise is ineffective in nonhypoxic COPD patients. Eur Respir $J$ 2003;22:584-8.

22 O'Donnell DE, Revill SM, Webb KA. Dynamic hyperinflation and exercise intolerance in chronic obstructive pulmonary disease. Am J Respir Crit Care Med 2001; 164:770-7.

23 Eltayara L, Becklake MR, Volta CA, et al. Relationship between chronic dyspnea and expiratory flow limitation in patients with chronic obstructive pulmonary disease. Am J Respir Crit Care Med 1996;154:1726-34.

24 Koulouris NG, Dimopoulou I, Valta P, et al. Detection of expiratory flow limitation during exercise in COPD patients. J Appl Physiol 1997;82:723-31.

25 Spence DP, Graham DR, Ahmed J, et al. Does cold air affect exercise capacity and dyspnea in stable chronic obstructive pulmonary disease? Chest 1993;103:693-6. 